\title{
Aortic emergencies-diagnosis and treatment: a pictorial review
}

\author{
Esther Voitle • Wolfgang Hofmann • Manfred Cejna
}

Received: 24 July 2014 / Revised: 27 November 2014 / Accepted: 16 December 2014 / Published online: 1 February 2015

(C) The Author(s) 2015. This article is published with open access at Springerlink.com

\begin{abstract}
Objectives To demonstrate the various presentations of acute aortic pathology and to present diagnostic and therapeutic approaches.

Methods Diagnostic imaging is the key to the reliable diagnosis of acute aortic pathology with multi-slice computed tomography angiography (CTA) as the fastest and most robust modality. Endovascular aortic repair (EVAR) with stent grafts and open surgical repair are therapeutic approaches for aortic pathology.

Results CTA is reliable in diagnosing and grading aortic trauma, measuring aortic diameter in aortic aneurysms and detecting vascular wall pathology in acute aortic syndrome and aortic inflammation. CTA enables planning the optimal therapeutic approach. Stent graft implantation and/or an open surgical approach can address vascular wall pathology and exclude aortic aneurysms.

Conclusion Aortic emergencies have to be detected quickly. CTA is the imaging method of choice and helps to decide whether elective, urgent or emergent treatment is necessary with EVAR and open surgical repair as the main treatment approaches.

Teaching Points

- To present aortic pathology caused by trauma

- To present acute aortic syndrome (aortic dissection, intramural haematoma and penetrating ulcers)

- To present symptomatic and ruptured aortic aneurysm
\end{abstract}

E. Voitle $\cdot$ M. Cejna $(\bowtie)$

Institute for Diagnostic and Interventional Radiology, Academic

Teaching Hospital LKH Feldkirch, Carinagasse 47, 6800 Feldkirch,

Austria

e-mail: manfred.cejna@1khf.at

W. Hofmann

Department of Vascular Surgery, Academic Teaching Hospital LKH

Feldkirch, Carinagasse 47, 6800 Feldkirch, Austria
- To present infection (mycotic aneurysms/aorto-duodenal fistulae) or iatrogenic injury of the aorta

- To understand different presentations for treatment planning (EVAR and open surgery)

Keywords Aortic pathology · Aortic Dissection · Aortic Aneurysm $\cdot$ Diagnostic imaging $\cdot$ Endovascular aortic repair (EVAR)

\section{Introduction}

Aortic emergencies present a diagnostic and treatment challenge for emergency physicians [1].

Aortic emergencies comprise different clinical entities with only one thing in common: if not diagnosed and managed in due time they are life threatening [2-5]. Compared to myocardial infarction and stroke, aortic pathologies are relatively rare (Table 1). A rapid diagnosis and treatment decision is therefore necessary to decrease mortality. CTA has become the best modality for the diagnosis and treatment planning of aortic pathologies $[3,4,6,7]$. Treatment is performed by either an open surgical approach or endovascular stent graft implantation (endovascular aortic repair,EVAR) [3, 7-9].

\section{Computed tomography imaging}

Currently, the most often used imaging modality in emergency radiology is computed tomography $(\mathrm{CT})$ imaging $[3,6,10$, 11]. It is a robust, readily available modality in most hospitals. CT scanners are often located in close proximity to the trauma or emergency rooms. With the introduction of 16-slice scanners iso-volumetric imaging was possible, enabling 3D and multi-axial imaging. Sixteen-slice scanner examinations are either fast (larger collimations $>1 \mathrm{~mm}$ ) or have a high z-axis 
Table 1 Incidences of various aortic pathologies compared to myocardial infarction and stroke

\begin{tabular}{|c|c|}
\hline & Incidence \\
\hline Myocardial infarction & $\begin{array}{l}80-330 \text { patients } / 100,000 \text { patients/year at the } \\
\text { age of } 45-54 \text { and } 350-1,140 \text { patients/ } \\
100,000 \text { patients/year at the age of } 65-74 \text { [2] }\end{array}$ \\
\hline Stroke & $180-294$ patients/100,000 patients/year [2] \\
\hline Acute aortic syndrome & $\begin{array}{l}\text { The majority of patients present with aortic } \\
\text { dissection (AD); its incidence is estimated to } \\
\text { be } 2-3.5 \text { patients/100,000 patients/year [3]. } \\
\text { Accurate numbers are difficult to obtain } \\
\text { because just like in the case of ruptured } \\
\text { aneurysms many patients may die without } \\
\text { proper diagnosis, without reaching a hospital } \\
\text { and/or receiving treatment }\end{array}$ \\
\hline $\begin{array}{l}\text { Thoracic aortic } \\
\text { aneurysm }\end{array}$ & $\begin{array}{l}\text { The incidence of thoracic aortic aneurysms is } \\
\text { approximately } 10 \text { cases per } 100,000 \text { person- } \\
\text { years [4], estimated to be increasing }\end{array}$ \\
\hline $\begin{array}{l}\text { Abdominal aortic } \\
\text { aneurysm }\end{array}$ & $\begin{array}{l}\text { In the abdominal aorta aneurysms can be found } \\
\text { in as many as } 7 \% \text { of patients when } \\
\text { screening in the }>65 \text {-year population [5]. } \\
\text { Studies showed the highest prevalence of } \\
\text { AAA }>3.0 \mathrm{~cm} \text { was } 5.9 \% \text { and was found in } \\
\text { white male smokers between } 50 \text { and } \\
79 \text { years [6] }\end{array}$ \\
\hline $\begin{array}{l}\text { Symptomatic aneurysm } \\
\quad \text { (abdominal) }\end{array}$ & $\begin{array}{l}\text { Symptomatic AAA in males has an incidence } \\
\text { of } 25 \text { per } 100,000 \text { at age } 50 \text {, increasing to } 78 \\
\text { per } 100,000 \text { over the age of } 70 \text { [7] }\end{array}$ \\
\hline $\begin{array}{l}\text { Ruptured aneurysm } \\
\text { (abdominal) }\end{array}$ & $\begin{array}{l}\text { The incidence of ruptured abdominal aortic } \\
\text { aneurysms ranges between } 5.6 \text { and } 17.5 \text { per } \\
100,000 \text { person-years in Western countries } \\
\text { [7]. The incidence of AAA is estimated to be } \\
\text { decreasing, as well as the incidence of AAA } \\
\text { rupture [7] }\end{array}$ \\
\hline $\begin{array}{l}\text { Aorto-enteric fistulae } \\
\text { (AEF) }\end{array}$ & $\begin{array}{l}\text { AEFs are very rare with an incidence of } 0.1 / \\
100,000 \text { patients/year, mostly secondary } \\
\text { aorto-enteric fistulae in patients with a } \\
\text { history of aortic aneurysm and/or surgical } \\
\text { aortic repair. Primary aorto-enteric fistulae } \\
\text { without previous surgical aortic repair have } \\
\text { an even lower incidence }\end{array}$ \\
\hline
\end{tabular}

resolution (for collimations from 0.5 to $0.75 \mathrm{~mm}$ ). The 64 slice scanners combine both fast imaging and high z-axis resolution. Dual energy and/or 256-320-slice scanners do
Fig. 1 Grading of traumatic aortic injury in para-sagittal CT reformations with additional schematic presentation: (a) Grade 1 injury in a 38-yearold patient after a fall from $10 \mathrm{~m}$. Intimal flaps are demonstrated at the level of the upper and lower curvature of the proximal descending aorta and $10 \mathrm{~cm}$ distal to the subclavian artery (arrowheads) and accompanying mediastinal haematoma. (b) Grade 3 injury in a 35-year-old female patient after a motor vehicle accident. A large pseudoaneurysm formation is seen in the typical position. (c) and (d) Traumatic aortic transection (grade 4) in a 79-year-old female patient after a fall from $4 \mathrm{~m}$ height. There is also a massive para-aortic haematoma. The patient died immediately after the CT examination

not significantly increase z-axis resolution but are faster than 64-slice scanners. Imaging of the whole aorta $(48 \mathrm{~cm})$ may take $20 \mathrm{~s}$ (16-slice CT with small collimation), $10 \mathrm{~s}$ (16-slice CT with large collimation), $6 \mathrm{~s}$ (for 64-slice CT) or well below $3 \mathrm{~s}$ (for 256-320-slice or dual CT).

CT is unsurpassed in its ability to rapidly detect aortic pathology, sometimes already in non-contrasted scans (e.g., aneurysm rupture or intramural haematoma). However, non-contrast scans are not always necessary for diagnosis; multiphasic scans multiply the total radiation burden. CT angiography is usually reserved for true arterial phase CT. All aortic pathologies or associated pathologies are easily depicted in both the arterial and the delayed (venous) phase. Delayed phases are often included in an imaging protocol (in addition to CTA) to clearly depict organ malperfusion (in dissections) or identify additional venous or organ bleeding (in trauma). Table 2 shows imaging protocols for examination of different aortic pathologies.

Fast imaging, quick image reconstruction and image transfer to the workstation and fast (3D) image processing are the main advantages of modern CT systems in emergency radiology. 3D vascular reconstruction with vascular segmentation and centreline reconstructions are often helpful in aortic measurements for stent graft planning. This is easier to perform in the arterial phase than in the venous phase.

The drawbacks of $\mathrm{CT}$ are the use of contrast media and ionizing radiation. Contrast media may pose a threat of worsening of renal function in patients with chronic renal insufficiency, although CTA imaging of the whole aorta is possible
Table 2 Potential protocols with multiphasic CTA to depict aortic pathologies (optional or standard parts of a multiphasic protocol)

\begin{tabular}{llll}
\hline Suspicion of & Non-contrast scan & $\begin{array}{l}\text { CTA=Arterial phase } \\
\text { (bolus triggered })\end{array}$ & $\begin{array}{l}\text { Delayed (venous) phase } \\
(+20-30 \text { s after CTA })\end{array}$ \\
\hline Aortic trauma & Optional & Standard & Standard \\
Aneurysm/aneurysm rupture & Optional & Standard & Optional \\
Aortic dissection & Recommended & Standard & Standard \\
Intramural haematoma & Recommended & Standard & (Standard) \\
Penetrating aortic ulcer & Recommended & Standard & (Standard) \\
Aorto-enteric fistulae & Recommended & Standard & Standard \\
Aortitis & Optional & Standard & Standard \\
\hline
\end{tabular}



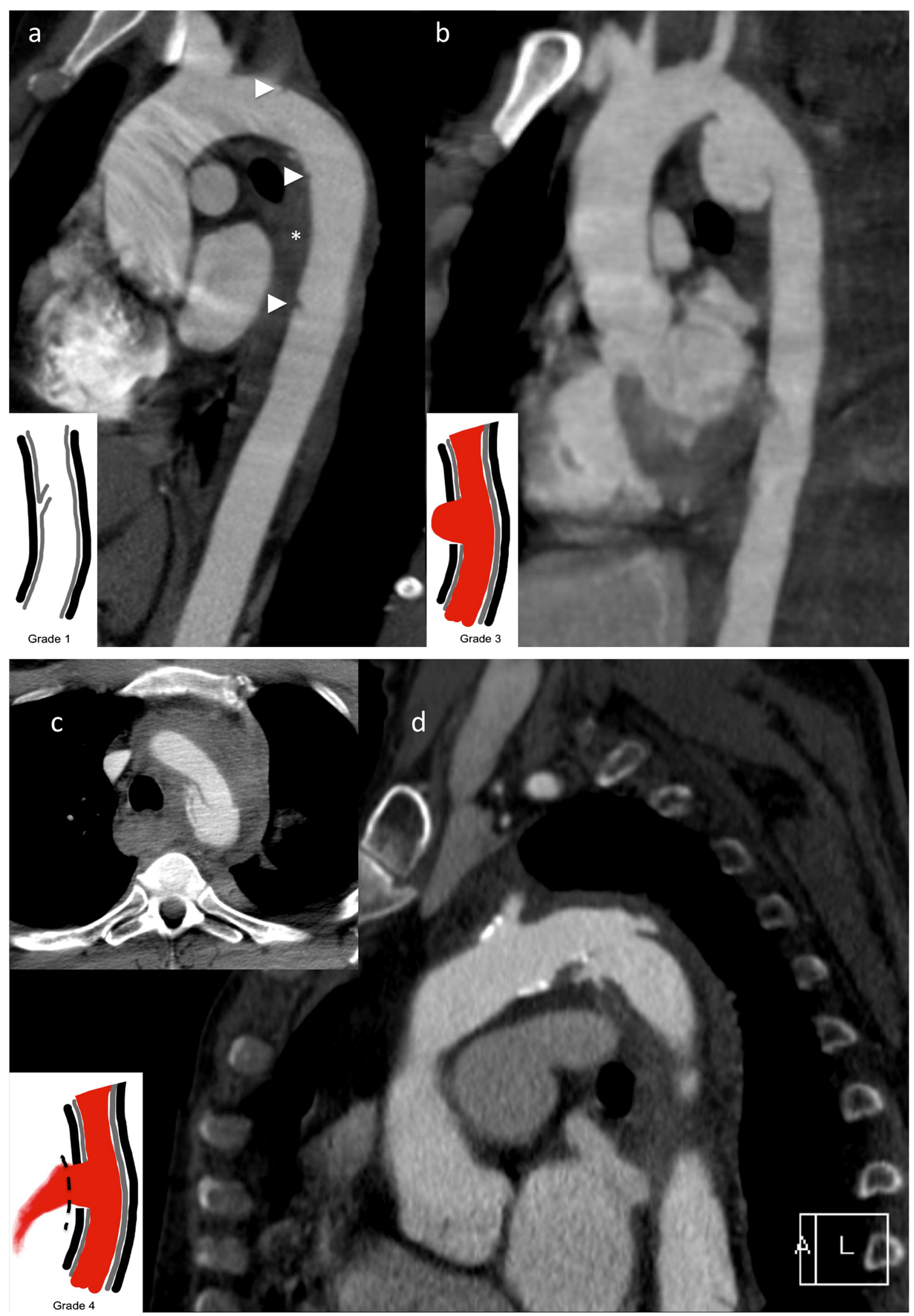
Fig. 2 Traumatic aortic injury with stent graft implantation. A 49-year-old male patient presented with aortic trauma after a motor vehicle accident. An aortic transection with a grade 3 lesion of the proximal descending aorta (a) and accompanying mediastinal haematoma (b) is seen on a para-sagittal reformation. CTA and DSA demonstrate patency of both vertebral arteries. More pronounced pseudoaneurysm formation prior to stent graft placement and exclusion of the pseudoaneurysm after stent graft placement (e) over the left subclavian artery (arrowhead) that is still filling during control angiography

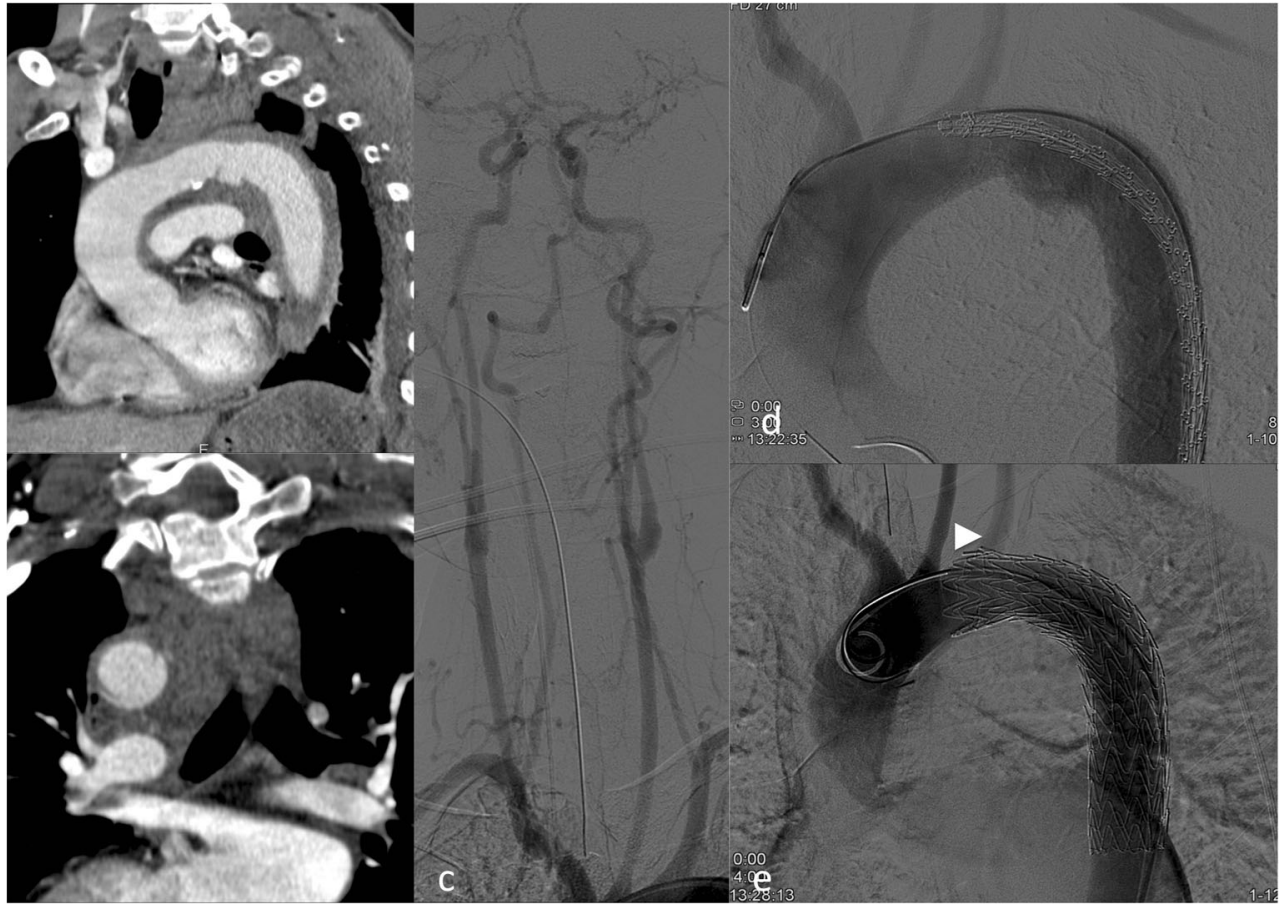

with as little as $40-50 \mathrm{ml}$ of contrast with 64 -slice systems (own observation).

Ionizing radiation poses a potential long-term risk especially in children and younger adults when CT is used to exclude pathology rather than to confirm it and excessive CT examination protocols are used. If CT is used for multiple follow-up examinations (EVAR, follow-up of aortic dissections) the radiation burden multiplies. In those instances the omissions of iodinated contrast media and radiation exposure are the clear advantages of (contrast-enhanced) ultrasound in the abdominal aorta (AA) or magnetic resonance imaging in the abdominal or thoracic aorta (AA or TA).

\section{Traumatic aortic injury}

Traumatic aortic injury is a life-threatening consequence of major blunt thoracic trauma, with high early mortality if relevant trauma is untreated $[3,4]$. From a clinical standpoint traumatic aortic injury has to be excluded after a relevant trauma, especially when associated with hypotension, fractures including those of the spine, scapula long bone or pelvis, pulmonary contusion and haematothorax. CT angiography is the method of choice for diagnosing aortic injury after major trauma [3].

CT diagnosis relies on the confirmation of luminal irregularities (intimal flaps), outer contour irregularities (pseudoaneurysms) and periaortic haematoma, signs of contrast extravasation or frank transections of the aorta. The predilection site is the transition zone between the relatively free-moving proximal thoracic aorta (including the arch) and the fixed portion at the aortic isthmus immediately distal to the left subclavian artery. According to Azizzadeh et al. [12], and adapted by the Society for Vascular Surgery (SVS), traumatic aortic injury can be classified into four categories [12, 13]: grade I (intimal tear), grade II (intramural haematoma), grade III (pseudoaneurysm) and grade IV (rupture) (Fig. 1).

Patients with grade I can be treated conservatively [10]. Invasive treatment is currently advocated for the treatment of grades II-IV [13], with some authors recommending endovascular treatment in grade II lesions only in the presence of severe periaortic haematoma at the level of the aortic arch $[14,15]$. Sometimes a staged approach for aortic repair in lower traumatic aortic injury grades (grades II and III) can be considered, treating more severe injuries (head and brain, organ pathologies) prior to the aortic injury. Nevertheless short-term follow-up to exclude progressing pseudoaneurysm or haematoma formation is recommended.

Fig. 3 Type A dissection. A 56-year-old patient presented with chest pain in the emergency department. CTA demonstrated a type A dissection. The tear starts at the level of the sinotubular junction and extends into the supraaortic arteries (arrowhead) and the abdominal aorta. The false lumen opacifies to a lesser extent (and later) than the true lumen $(\mathbf{a}-\mathbf{c})$. The patient was treated with a modified Bentall procedure (composite graft replacement of the aortic valve, aortic root and ascending aorta, with re-implantation of the coronary arteries) (c-e). Ruptured type A dissection in a 71-year-old female patient (after implantation of a mechanical aortic valve). She presented with acute chest pain. CTA demonstrated a type A dissection restricted to the ascending aorta starting at the sinotubular junction [coronal (g) and sagittal (h) CT reformations]. The axial CT scans (I-j) demonstrate extravasation (arrowhead) and haematopericardium (asterisk) 

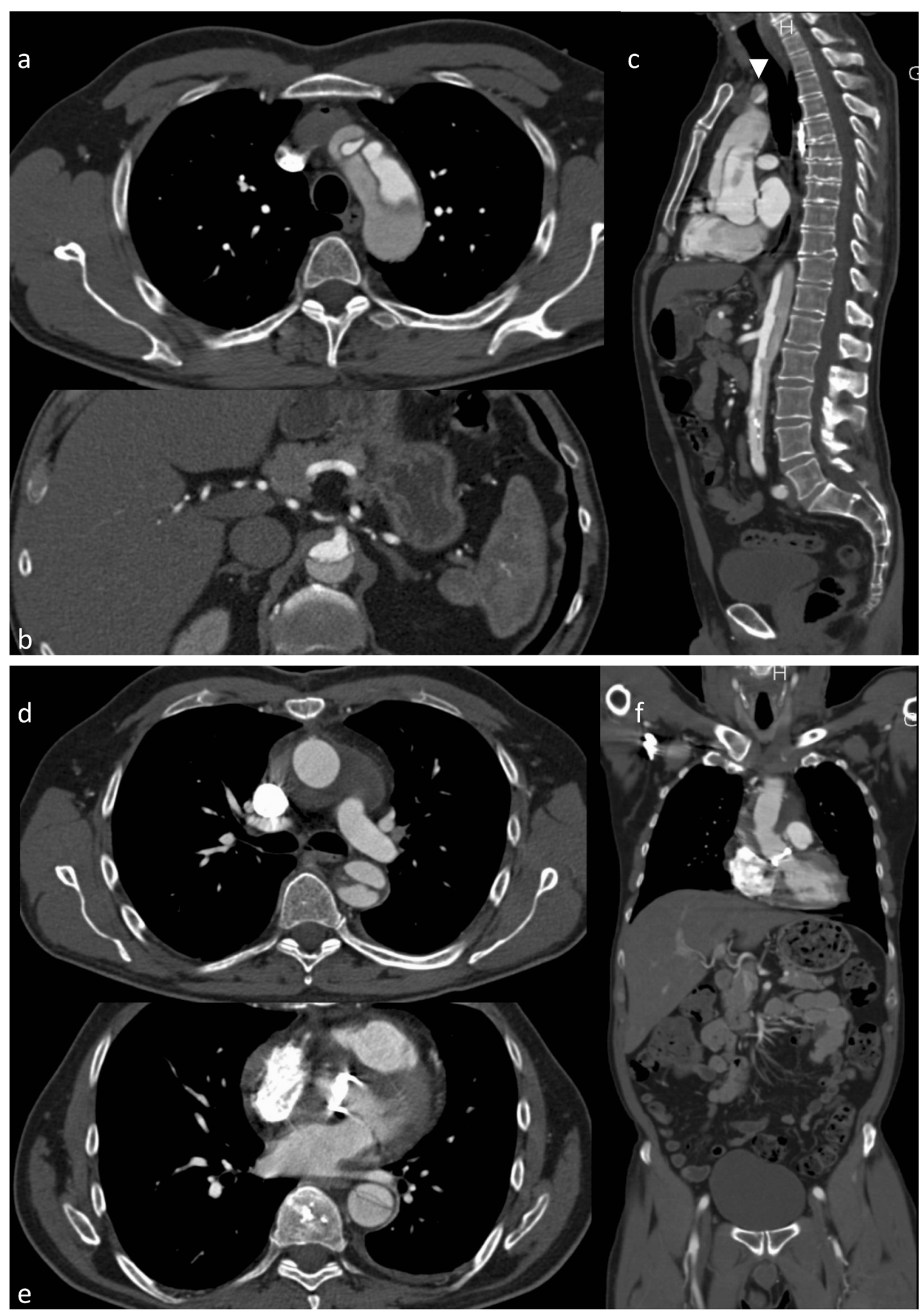


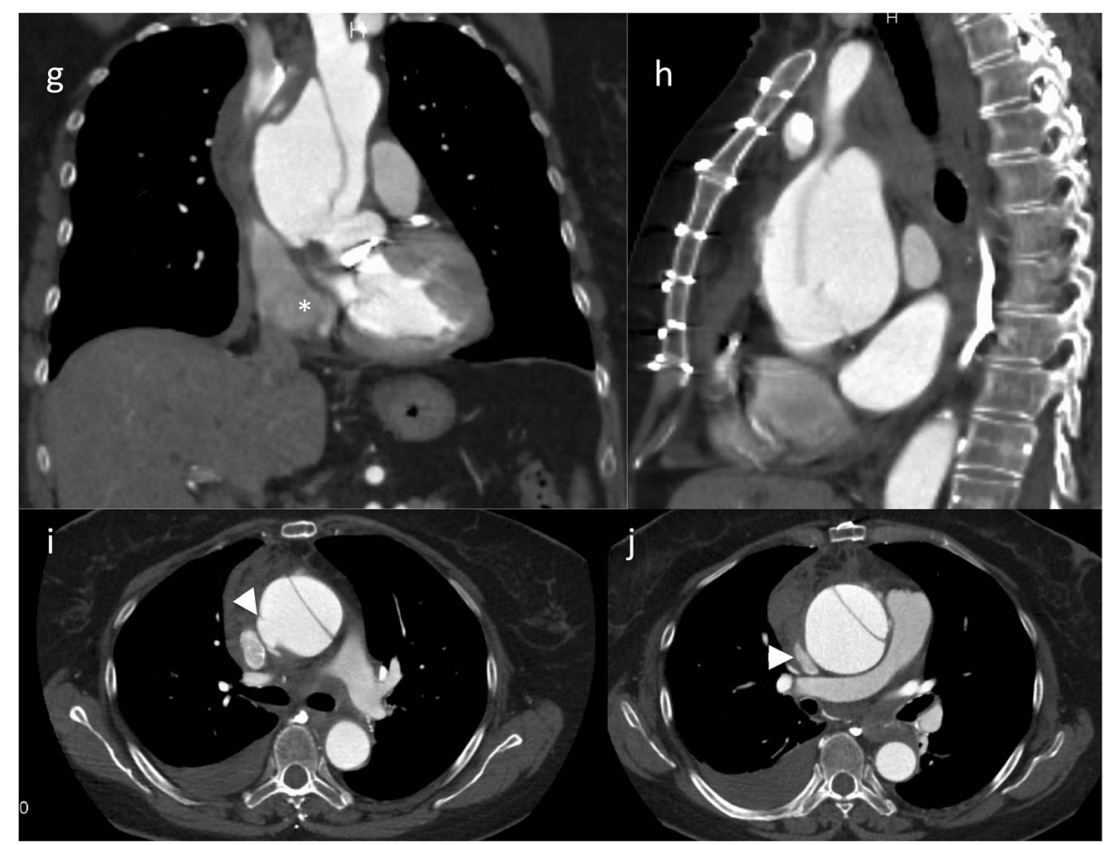

Fig. 3 (continued)

Endovascular treatment of thoracic aortic injuries is now the treatment of choice, reducing mortality and morbidity (e.g., paraplegia, renal insufficiency) compared to open surgical repair without sacrificing long-term clinical success with 5 -year survival rates up to $87 \%[8,9]$.

Since the predilection site for aortic injury is at the aortic isthmus in proximity to the left subclavian artery, stent graft implantation in up to $30 \%$ of the cases will result in partial or complete coverage of the left subclavian artery [13]. Prior to coverage of the left subclavian artery patency of the right vertebral artery has to be confirmed by either initial arterial imaging up to the circle of Willis or intra-arterial angiography prior to stent graft insertion (Fig. 2). With a patent right vertebral artery of a normal calibre ischaemic complications after coverage of the left vertebral artery are very rare [16].

On centreline reconstructions it is easy to measure the length of the aortic pathology and to measure the distance from healthy to healthy vessel segments, necessary for treatment planning (= stent graft length). Correct measurement of the aortic diameter is not straightforward; the aortic diameter is dependent on the cardiac cycle (systole versus diastole) and presence or absence of hypovolaemic shock [17, 18]. This has to be taken into consideration, because stent graft oversizing of more than 15-20\% of the aortic diameter is not recommended and can lead to future complications such as retrograde dissection and stent graft collapse [17, 18].

After stent graft implantation regular CT follow-up has to be performed. How often and until when have still not been ideally determined [19]. Since the young population is usually more at risk for traumatic aortic injury the radiation burden will be substantial over time, so MRA might be a reasonable alternative to CT [20].

\section{Acute aortic syndrome}

Initially coined by Vilacosta and Roman [21] the term "acute aortic syndrome" comprises three different but related entities:

- Aortic dissection (AD)

- Intramural haematoma (IMH)

- Penetrating atherosclerotic ulcer (PAU)

In all of these presentations, initial disruption of the medial layer of the aorta is the starting point of the vascular wall pathology. All entities share similar clinical presentations, most often sudden severe thoracic or abdominal pain, as the initial presentation. Sometimes ruptured aortic aneurysms are included under acute aortic syndrome; in this article ruptured aortic aneurysms are included as a separate chapter.

\section{Aortic dissection}

An entrance tear from the lumen to the media allows blood flow into the vascular wall and therefore creates a false lumen, usually with additional communication tears between the old "true" and the new "false" lumen. The false lumen has no regular vessel wall, thus leading to aortic diameter enlargement, (pseudo) aneurysm formation and potential rupture 
Fig. 4 Ruptured type B dissection with stent graft implantation. An 83-year-old patient with ruptured type $B$ dissection in para-sagittal reformations demonstrating intimal tear (a) and haematothorax $(\mathbf{a}, \mathbf{b})$. Stent graft implantation (c) (partially covering the left subclavian artery) and exclusion of the dissection after expansion of the stent graft (d)

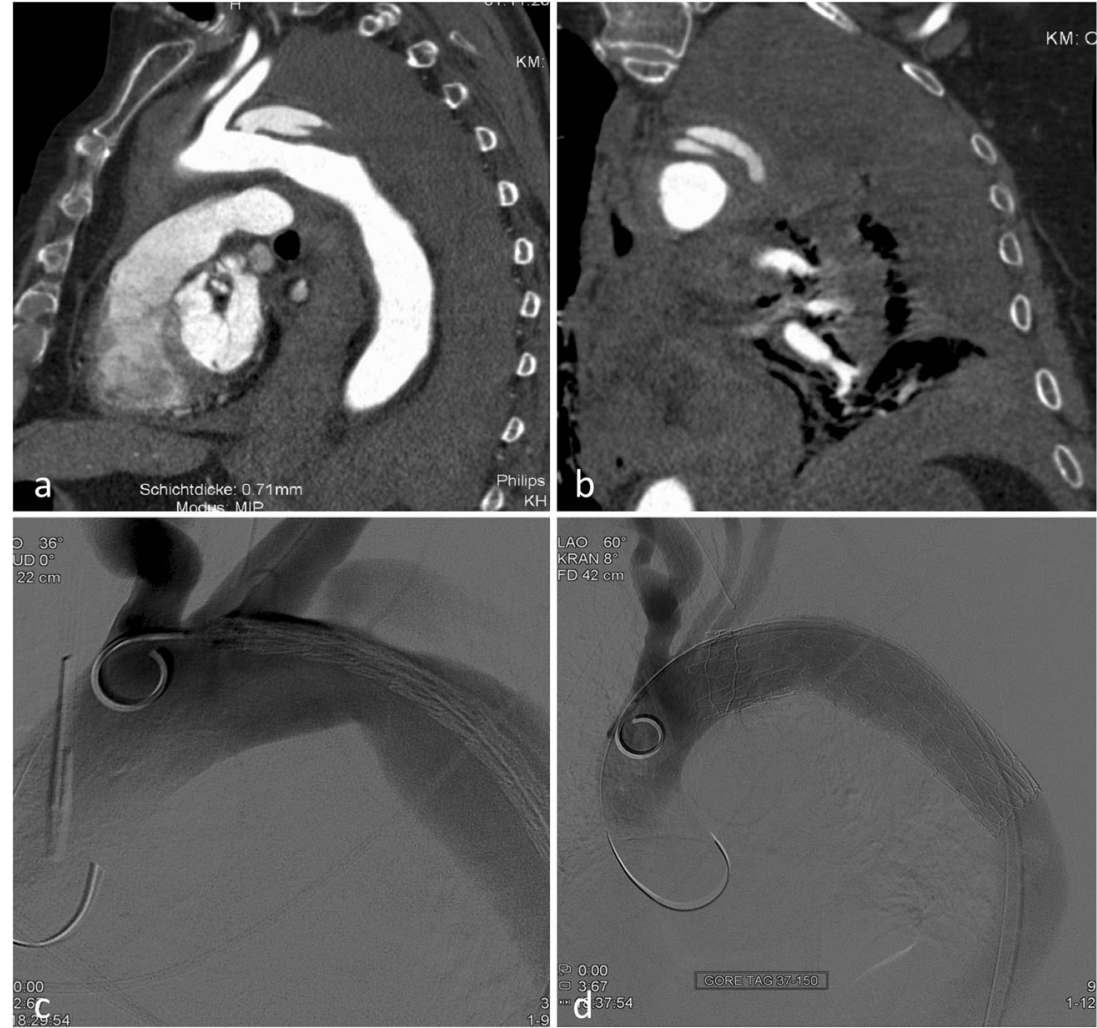

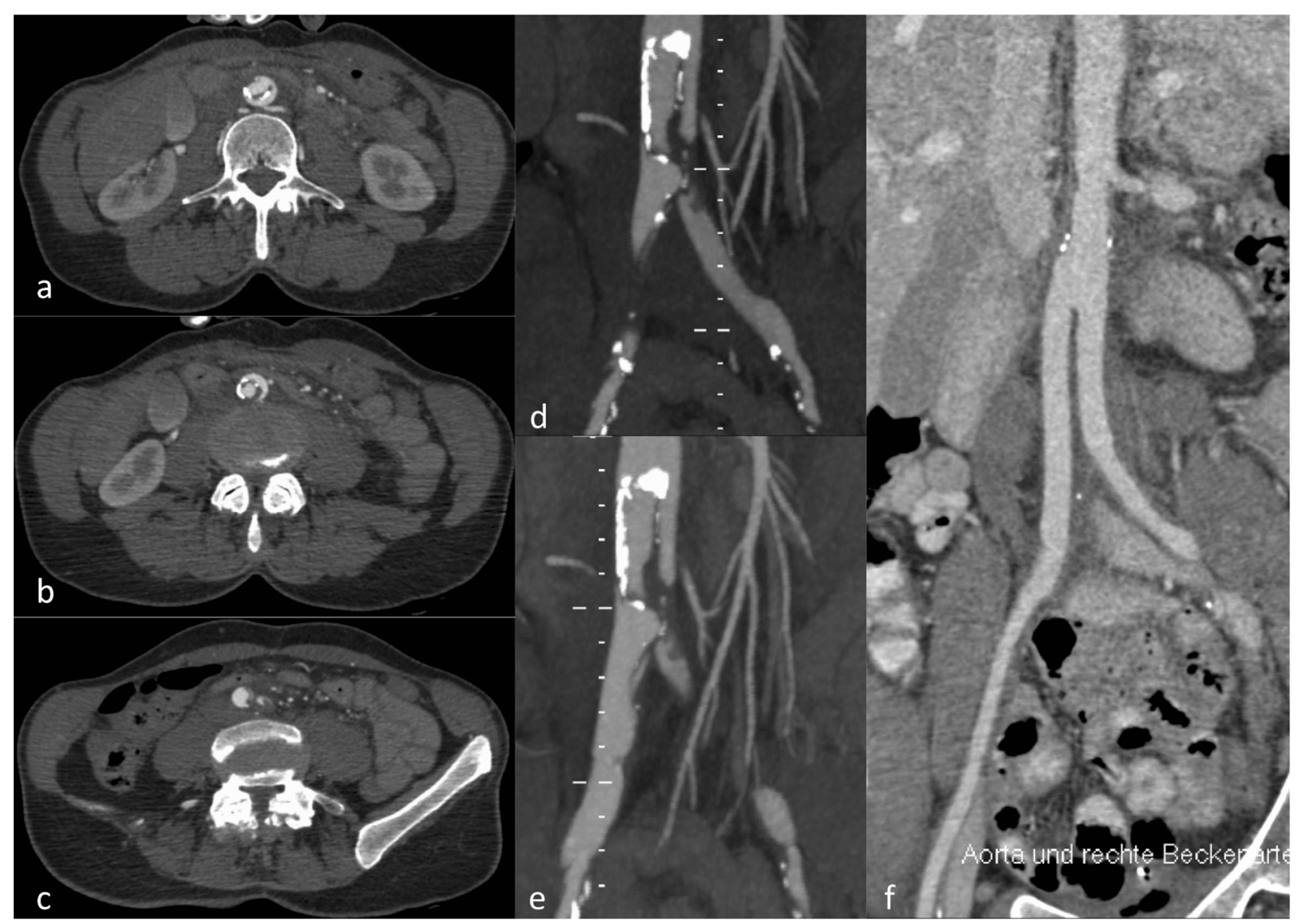

Fig. 5 Traumatic type B dissection in the infrarenal abdominal aorta in a 55 -year-old female patient who fell from $3 \mathrm{~m}$ height. The patient presented with abdominal pain and acute bilateral limb ischaemia caused by the dissection reaching into the common iliac artery on both sides (a-e). She was treated by a surgical approach with implantation of a bifurcated prosthesis (f) 
during short- and long-term follow-up. Classification of aortic dissection is based on the location and extension of the dissection (Stanford and DeBakey classifications $[3,4]$ ) predominantly based on involvement of the ascending versus descending versus the total aorta. The Stanford classification system grades dissections as type A dissection (in all dissections involving the ascending aorta regardless of the site of origin, surgical treatment is recommended [3, 4]; Fig. 3) or type $\mathrm{B}$ dissections (exclusive involvement of the descending aorta; Figs. 4 and 5).

The intimomedial (= dissection) flap separating the true from the false lumen presents either as a spiralling flap or a complete circumferential dissection. In aortic dissection it is necessary to distinguish between a true and false lumen.

The true lumen more often has a cylindrical or filiform shape. It is in clear continuity with the non-dissected proximal portion of the aorta (this is more difficult to demonstrate for type A dissections). In acute dissections it is the lumen that contains outer wall calcification. Usually the true lumen starts opacifying earlier in the arterial phase [22]. The false lumen has a characteristic "beak" appearance; this is an acute angle between the dissection flap and the outer wall of the false lumen. The false lumen starts opacifying later than the true lumen [22]. The use of retrospective gating (more radiation dose) or prospective gating (step and shoot with an option for dose-saving protocols) makes imaging of the entrance tear and re-entries (additional communications between the true and false lumen) easier than ungated CT [6].

Recently a new and more complex mnemonic-based classification system has been introduced by Dake et al. [23]; the word DISSECT takes several clinical and imaging key factors of aortic dissection into account and therefore helps in treatment planning (medical versus endovascular versus open surgical repair):

- Duration of disease (acute for symptoms $<14$ days, subacute $=14$ days to 3 months and chronic for $>3$ months). A marked curvature of a mobile dissection flap is more characteristic of acute dissections, whereas a rather flat and fixed appearance of an immobile and thickened flap (fibrosis leading to reduced flap mobility) is more characteristic of a chronic dissection.

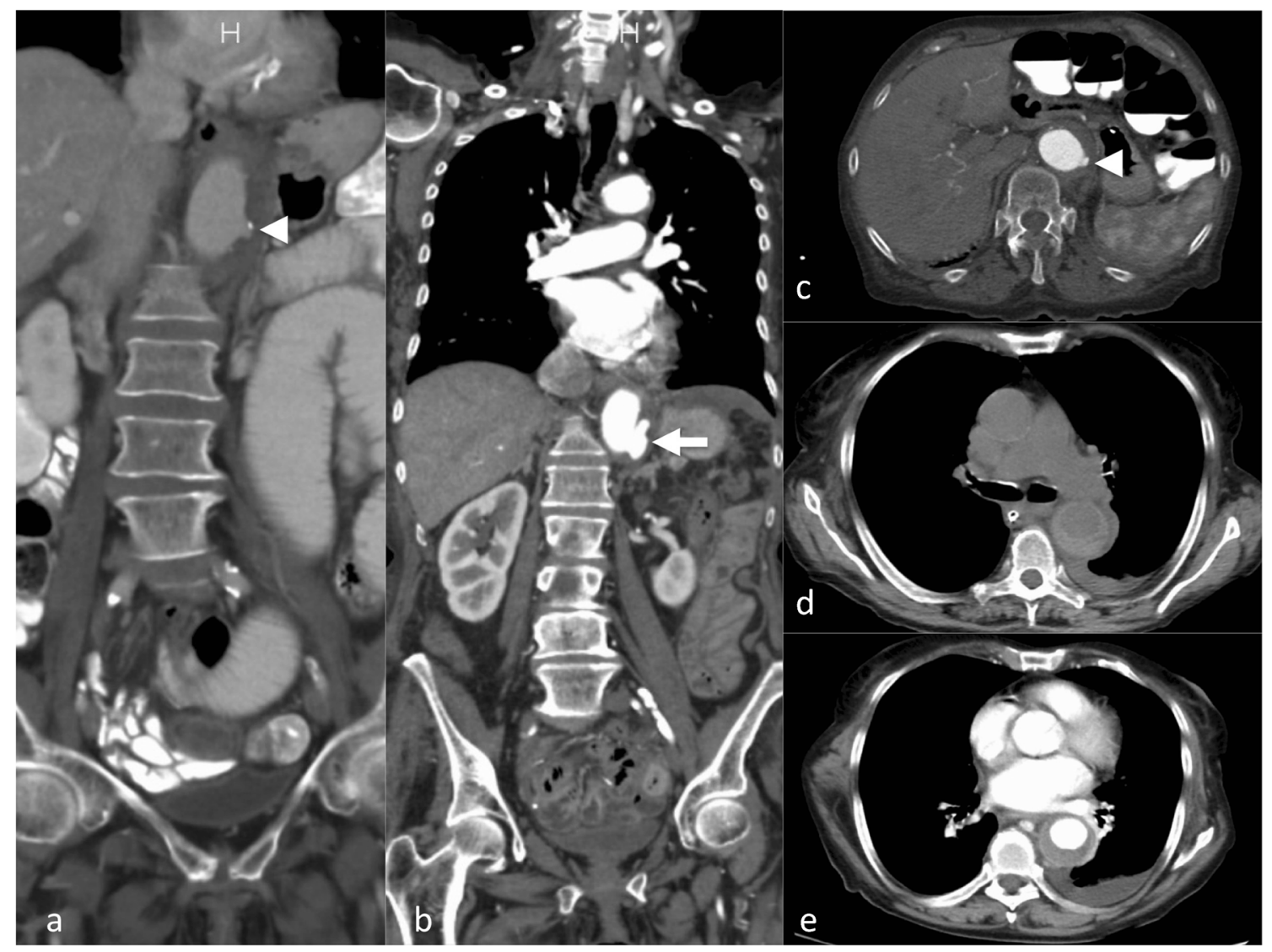

Fig. 6 Intramural haematoma in a 79-year-old female patient. The patient presented with thoracic pain. Initial CTA with coronal reformations (a) and axial section (c) CTA demonstrated a small ulcerlike configuration (arrowhead) in the intramural haematoma that ascended up to the left subclavian artery (a). One-week follow-up CTA demonstrated enlargement of the ulcer-like configuration (arrow) (b). Native CT demonstrates the hyperdense intramural haematoma (d) and contrast-enhanced $\mathrm{CT}$ the smooth circumferential configuration of the intramural haematoma (e) 
- Intimal tear location (also takes retrograde dissections into consideration, for example a type A dissection with an entrance tear in the descending aorta distal to the subclavian artery).
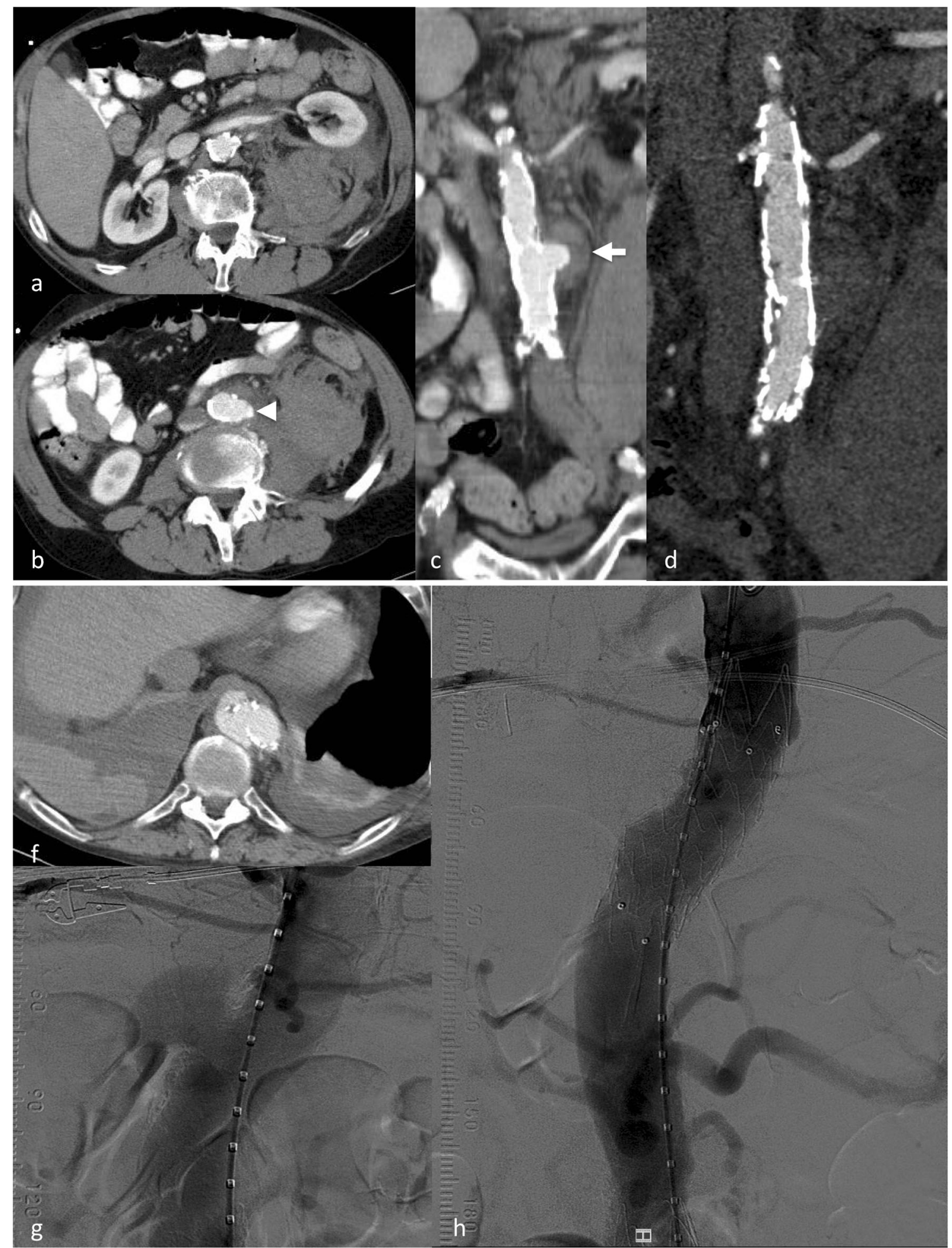

Fig. 7 Ruptured penetrating aortic ulcer in a 57-year-old male patient. Chronic renal failure led to haemodialysis. The patient presented with hypotension and abdominal pain. CTA demonstrated a large retroperitoneal haematoma (a) and an aortic ulcer projecting beyond the aortic contour (arrowhead) (b). Coronal reformation demonstrated the mushroom-like appearance of the ulcer (arrow) (c). The patient was treated with implantation of a uni-iliac stent graft (d). Three years later he presented with hypotension and thoracic pain. CTA demonstrated a ruptured aortic ulcer in the distal descending aorta (e) that was again treated with implantation of a thoracic stent graft (f-g) 
- Size of the dissected aorta (in $\mathrm{mm}$ ), rapid growth or a diameter exceeding $50 \mathrm{~mm}$ during follow-up warranting invasive treatment.

- Segmental Extent (extent of aortic involvement from proximal to distal). For abdominal aortic dissection a surgical approach has some advantages because it is easier to manage the proximity and involvement of renal, visceral and peripheral (iliac) arteries (Fig. 6). The choice for surgery or stent grafting depends on the location and extent (thoracic only versus thoraco-abdominal or abdominal). Usually an open surgical approach in thoracic aorta dissection has higher morbidity and mortality than EVAR.

- Clinical complications of the dissection (complicated versus uncomplicated dissection). A complicated type B dissection is either categorised by refractory hypertension (and pain) and/or malperfusion syndrome (in $10 \%$ spinal, iliac and visceral arteries may be involved, clinical signs are often difficult to detect, and CT is necessary for early diagnosis) and/or haemodynamic instability ( $<90 \mathrm{mmHg}$ systolic or at shock) and/or peri-aortic haematoma and haemorrhagic pleural effusion (with an increase on two subsequent CTs) and/or rupture or signs of impending rupture. Stent graft implantation or open surgery is usually reserved for symptomatic or complicated dissections [5] (Figs. 4 and 5). For uncomplicated dissections usually a conservative treatment approach is used. Follow-up imaging is necessary to rule out a diameter increase, pseudoaneurysm formation or progression of the dissection.

- Thrombus (thrombosis) within the aortic false lumen. Complete false lumen thrombosis is the key to long-term clinical success and long-term survival.

\section{Intramural haematoma (IMH)}

The concept of IMH has changed over time [21, 24]. Initially it was defined as acute haemorrhage in the aortic wall, without apparent wall tear. This concept was updated after imaging [CTA, MRT or intravascular ultrasound (IVUS)] was able to depict wall tears causing the aortic wall haematoma [11]. The intramural haematoma formation is spontaneous and may be a consequence of trauma or a penetrating aortic ulcer (Fig. 6). It has been reported that IMH may progress to frank aortic dissection over time.

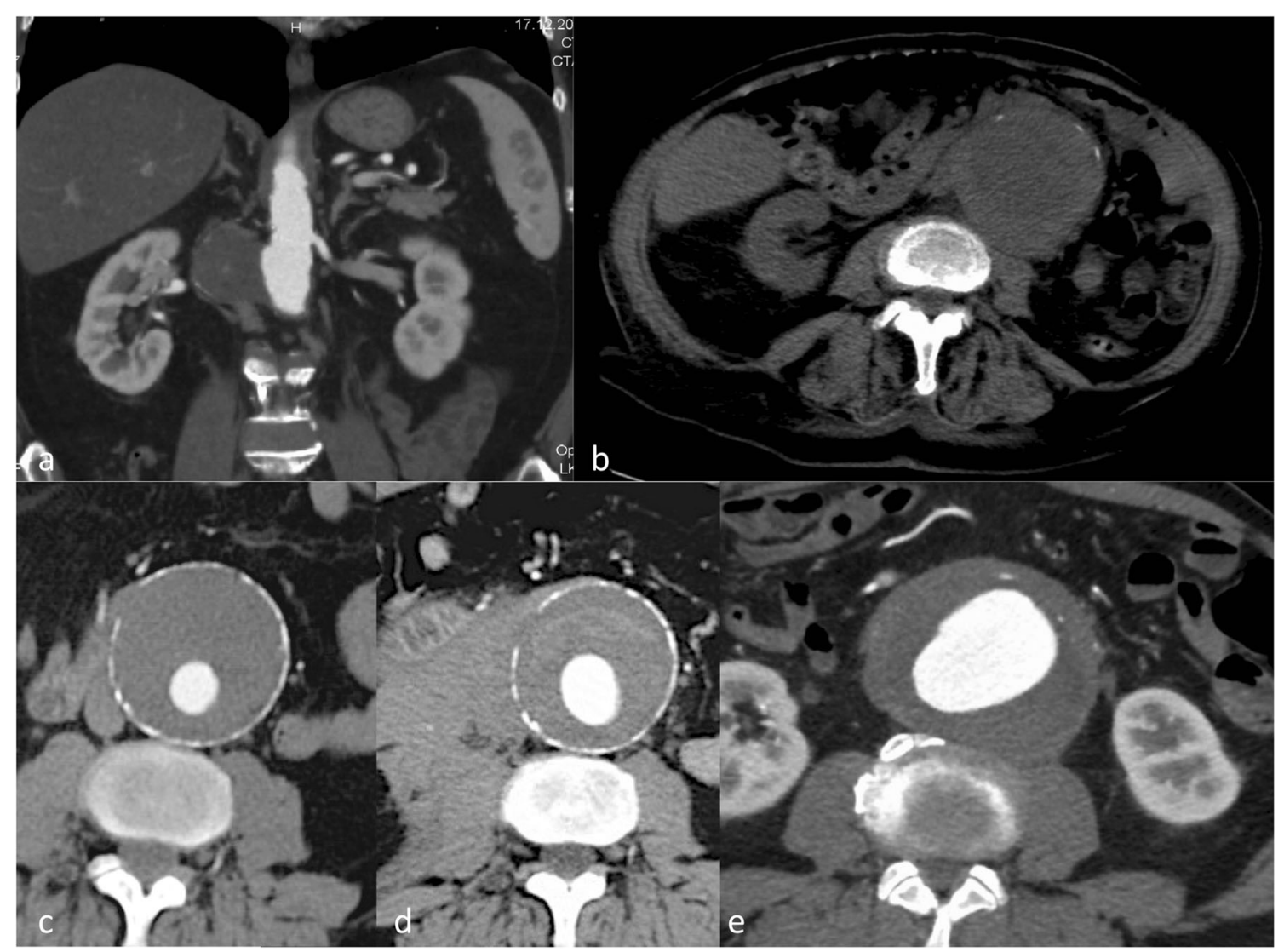

Fig. 8 Signs of impending rupture. Focal "blebbing" (arrow) of the aortic wall (a, c). "Crescent sign" with hyperdense thrombus (b). Draped aorta signs $(\mathbf{b}, \mathbf{e})$ with flattening of the posterior aspect of the abdominal aorta. Focal "discontinuation" of the aortic contour (here with discontinuation of the wall calcification and blebbing) (c) in a 6-cm abdominal aortic aneurysm. The patient refused further treatment. $\mathrm{He}$ presented with hypotension 3 months later. CTA demonstrated a ruptured aneurysm (d) 
Non-contrast images are very helpful in depicting the more hyperdense intramural haematoma (compared to the hypodense lumen area) but not essential. In CTA the circumferential intramural haematoma also has a very characteristic appearance (Fig. 6). Indications for endovascular treatment of IMH are basically the same as for dissection [25], namely symptomatic presentation, diameter increase, pseudoaneurysm formation or progression into AD during follow-up. Imaging aims to detect the entrance tear and EVAR aims to close the entrance tear, with complete stent graft coverage of the IMH having the best therapeutic outcome.

\section{Penetrating aortic ulcer (PAU)}

PAUs are most often found in patients with severe atheromatosis of the aorta or severe atherosclerosis, usually elderly patients with a multitude of co-morbidities. Atherosclerotic ulcers progress and erode the internal elastic membrane, paving the path to progression to wall haematoma [26], leading to the classic mushroom appearance (Fig. 7). The clinical (pain) and imaging presentation (growth, associated haematoma) may be the factors indicating treatment to prevent bleeding beyond the outer wall of the aorta, associated with a high risk of rupture or progression to $\mathrm{IMH}$ and $\mathrm{AD}$ (Fig. 6) [27]. Therefore, it is critical to identify PAUs as early as possible and perform regular follow-up or treatment with stent graft implantation over the penetrating ulcer [25, 28] (Fig. 7). There is currently no clear cutoff for a PAU diameter (depth) or neck diameter that warrants treatment, in one publication a depth of $>20 \mathrm{~mm}$ or a neck $>10 \mathrm{~mm}$ was associated with higher complication rates [26].

\section{Ruptured aortic aneurysm}

Diagnosis of aneurysms depends on measurement of the vascular diameter. The normal aortic diameter is dependent on location and age. For the ascending aortic diameter, the upper limit of the normal aorta is $35 \mathrm{~mm}$ at the age of 20; in an 80year-old patient the upper limit is close to $45 \mathrm{~mm}$ [4]. In the abdominal aorta, aneurysms are defined by a diameter $>30 \mathrm{~mm}$, regardless of the age of the patient. Aneurysms occur in isolation in the thoracic or abdominal aorta or can present as thoracoabdominal aortic aneurysms. The size of the aneurysm defines the rupture risk with a clear correlation between aneurysm diameter and the risk of rupture. For AAA the annual rupture risk is $1-11 \%$ for aneurysms between 50 and $59 \mathrm{~mm}, 10-22 \%$ for aneurysms sized from 60 to $69 \mathrm{~mm}$ and peak with rupture rates of 30-33\% for aneurysms larger than $70 \mathrm{~mm}$ [7].

There are definitive signs of impending rupture (an aneurysm size increase especially with a rapid enlargement rate, focal wall discontinuity, hyper-attenuating crescent sign,
Fig. 9 Ruptured abdominal aortic aneurysm with stent graft treatment. The 72-year-old male patient presented with left-sided abdominal pain and hypotension. CTA demonstrated a ruptured infrarenal aortic aneurysm on coronal and axial sections (a-b). The patient was treated with aorto-uni-iliac stent graft implantation (d) to the left, a crossover bypass and placement of an occluder (arrow) in the right common iliac artery. Follow-up CTA (c) demonstrated complete exclusion of the large aneurysm

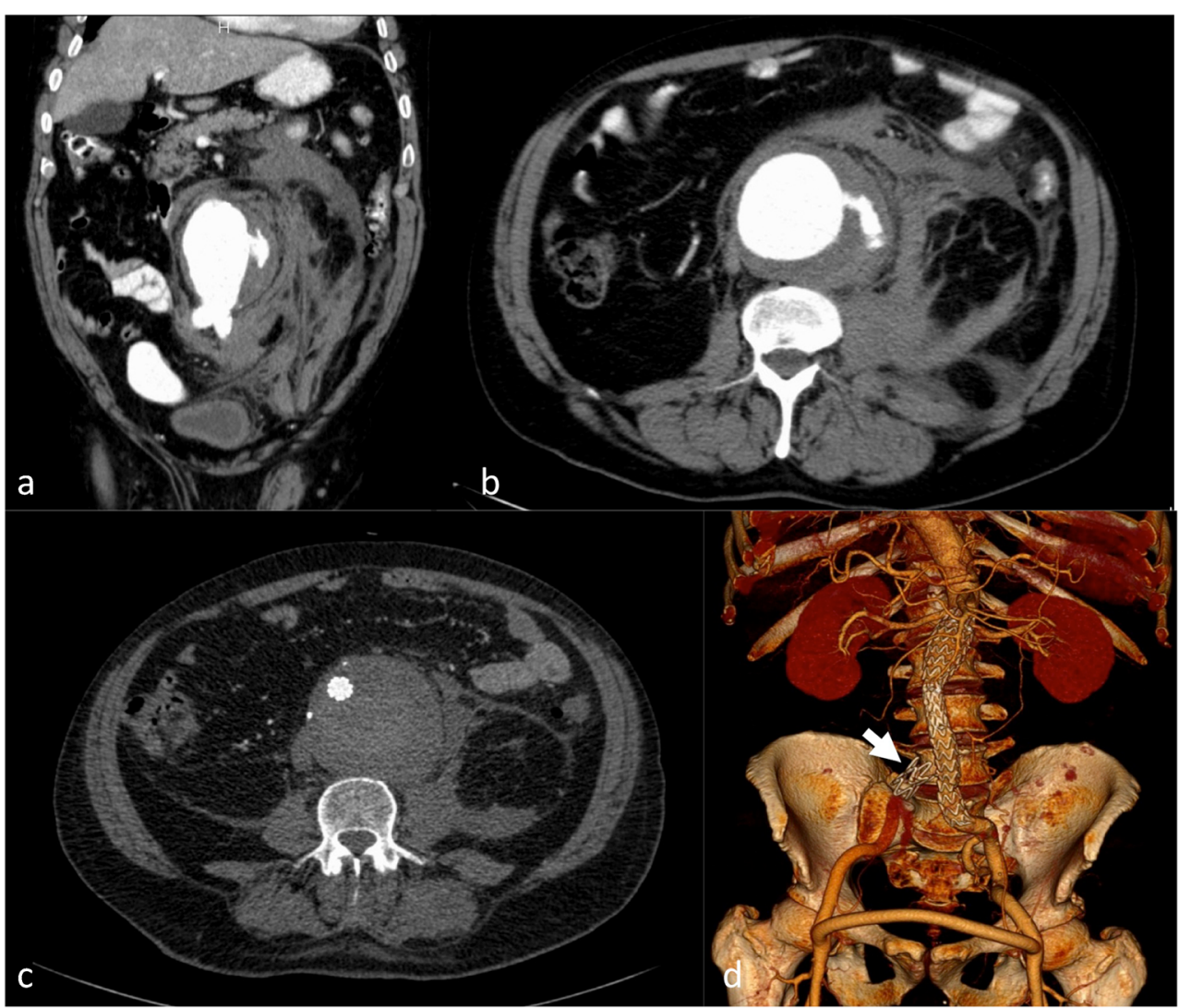


thrombus fissuration, draped aorta sign or periaortic stranding); unfortunately these signs are often not highly specific $[29,30]$ (Fig. 8) but detection of these findings advocates urgent treatment.

Imaging signs of completed rupture are contrast extravasation, retroperitoneal or intraperitoneal haematoma (Fig. 10), aorto-enteric or aorto-caval fistulae. AAA rupture can present as a contained rupture or free rupture. Patients with contained rupture should be managed with permissive hypotension to prevent a free rupture and keep the patient stable until treatment [31]. A free rupture can occur into the peritoneal cavity or retroperitoneal rupture with tissue tamponade reducing blood loss.

Clinical management consists of screening [especially in males (previous smokers) over 65 years], with yearly ultrasound surveillance for sizes of $30-45 \mathrm{~mm}$, referral to vascular centres with CTA for aneurysms $>45 \mathrm{~mm}$ and shorter followup times. Treatment is advocated when the rupture risk exceeds the risk of treatment. Currently, invasive treatment is recommended for infrarenal aortic aneurysm sizes $>55 \mathrm{~mm}$ or upon a diameter progression of $>0.5 \mathrm{~cm} /$ year [7]. In AAA, EVAR needs a suitable anatomy with infrarenal neck length
$>10 \mathrm{~mm}$ and neck diameters $<32 \mathrm{~mm}$, an appropriate diameter of access vessels (usually $>6 \mathrm{~mm}$ ) and absence of severe kinking in access vessels or in the infrarenal neck $\left(<60^{\circ}\right)$ for most of the available stent graft types. In the ascending aorta including the aortic arch, descending aorta and thoracoabdominal aneurysms, the cutoff for treatment is a diameter of more than $6 \mathrm{~cm}[4,7]$.

Symptomatic AAA is difficult to identify, detect and manage. The presenting symptoms are after non-specific abdominal pain, back pain or embolic events. When an AAA is demonstrated on imaging (regardless of size) and all other causes of abdominal pain or back pain have been ruled out (nephrolithiasis, diverticulitis, disk disease, etc.), this AAA can be considered symptomatic. Symptomatic aneurysms are thought to have a higher rupture risk than asymptomatic aneurysms; urgent surgical or endovascular repair is advocated.

The role of EVAR for the treatment of ruptured AAA (Fig. 9) is not completely clear. Approximately $50 \%$ of all patients with ruptured infrarenal aortic aneurysms are suitable for EVAR. EVAR was considered to have short- and long-time survival advantages over open surgery, but this was not confirmed in a large randomised trial [32].

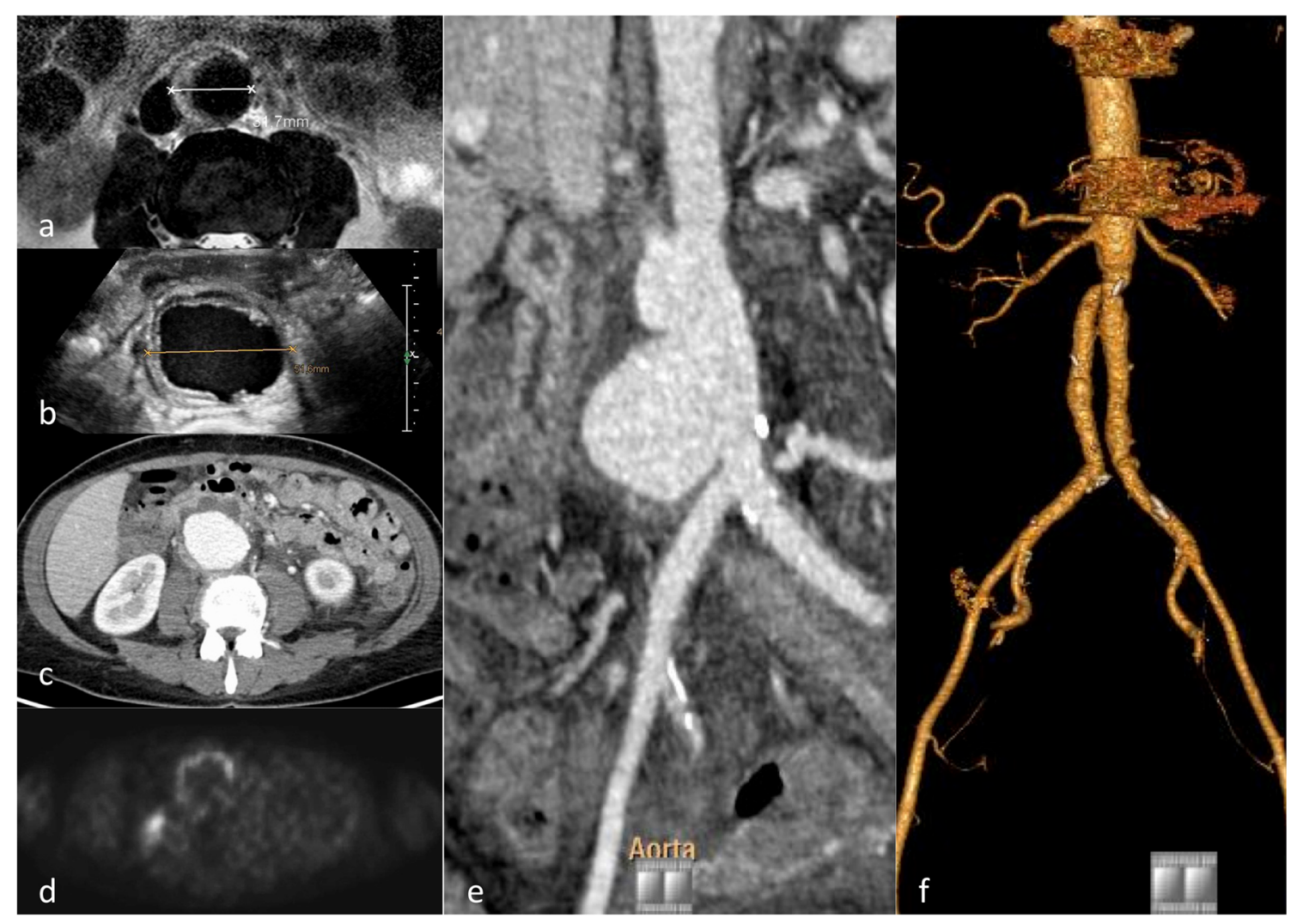

Fig. 10 Mycotic abdominal aortic aneurysm. The 51-year-old female patient presented with abdominal/lumbar discomfort and sub-febrile temperatures. A lumbar MRI demonstrated no spondylodiscitis and a small abdominal aortic aneurysm with $35 \mathrm{~mm}$ diameter. One month later the abdominal aortic diameter had grown to $52 \mathrm{~mm}(\mathbf{b}-\mathbf{c})$ and PET-CT (c-e) demonstrated wall thickening and PET uptake in the aortic wall and an atypical eccentric aneurysm presentation in coronal reformations (e). This was the only spot of infectious arthritis. Endocarditis of the mitral valve was demonstrated to be the focus. Additionally infectious arthritis of the left AC joint was treated by resection. Surgical reconstruction with deep vein (f) was performed; surgery confirmed a contained rupture and an aorto-duodenal fistulae 


\section{Mycotic aneurysms-infected aneurysms}

Infection is a rare cause of aortic aneurysms. Currently the term "infected aneurysm" or "infectious aortitis" is preferred over the term "mycotic aneurysm" (originally used in 1885 by Sir William Osler) since the majority of infectious agents are non-fungal (Staphylococcus aureus, Salmonella, Pneumococcus and Escherichia coli being the most common grampositive and -negative pathogens). Mycotic aneurysms are rare (1-2\% of all aneurysms) but life threatening, usually detected when they become symptomatic. Infected aneurysms have an atypical morphological presentation, as saccular aneurysms, eccentric aneurysms or pseudoaneurysms, but they may also present as grotesque fusiform aneurysms (Fig. 10). Rapid enlargement and (PET-positive) aneurysm wall thickening and/or blurred soft-tissue structure with contrast uptake and/or abscess formation or retentions accompanying the aorta are key findings in imaging and diagnosis of infected aneurysms. Mortality after open surgery or EVAR is considerably higher than for the treatment of conventional aortic aneurysms [33, 34]. Since EVAR does not address the underlying pathology (local infection) it sometimes scores as a bridging therapy to open surgical repair; only few cases have been described where EVAR alone with long-term antibiotic treatment was a curative treatment. Only surgical treatment (resection) \pm extra-anatomic reconstruction is considered a definitive treatment but with relatively high morbidity and mortality.

\section{Aorto-enteric fistulae (AEF)}

Aorto-enteric or aorto-duodenal fistulae are rare but often fatal late complications of open repair (after graft implantation) or aneurysm infection $[35,36]$. The primary diagnosis of AEF remains difficult; the clinical presentation is often with massive gastrointestinal bleeding, starting with herald bleeds and leading to severe bleeding episodes with exsanguination.

Just like in infected aneurysms computed tomography is the diagnostic imaging of choice in the acute phase. CT imaging findings suggestive of aortic infections (air/gas in the thrombus) are confirmed by PET-CT (fluorodeoxyglucose positron emission computed tomography) to be of infectious

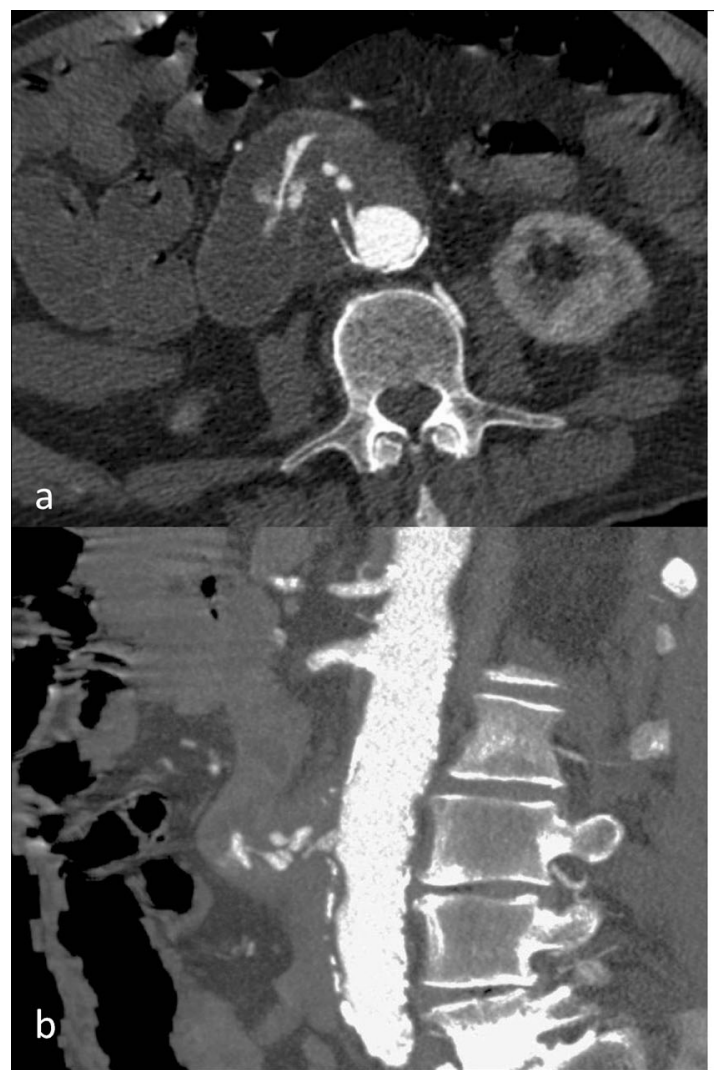

Fig. 11 Aorto-duodenal fistulae in a 75-year-old male patient presenting with melena and haematemesis. He had an aortic repair with a surgical tube graft 10 years ago. CTA (axial and coronal reformations) demonstrates contrast extravasation of the infrarenal aorta directly into the adjacent duodenum $(\mathbf{a}-\mathbf{b})$. The patient died immediately after the CT scan before stent graft implantation could be performed. In a 63 -year-old male patient with severe haematemesis ultrasound detected an aortic

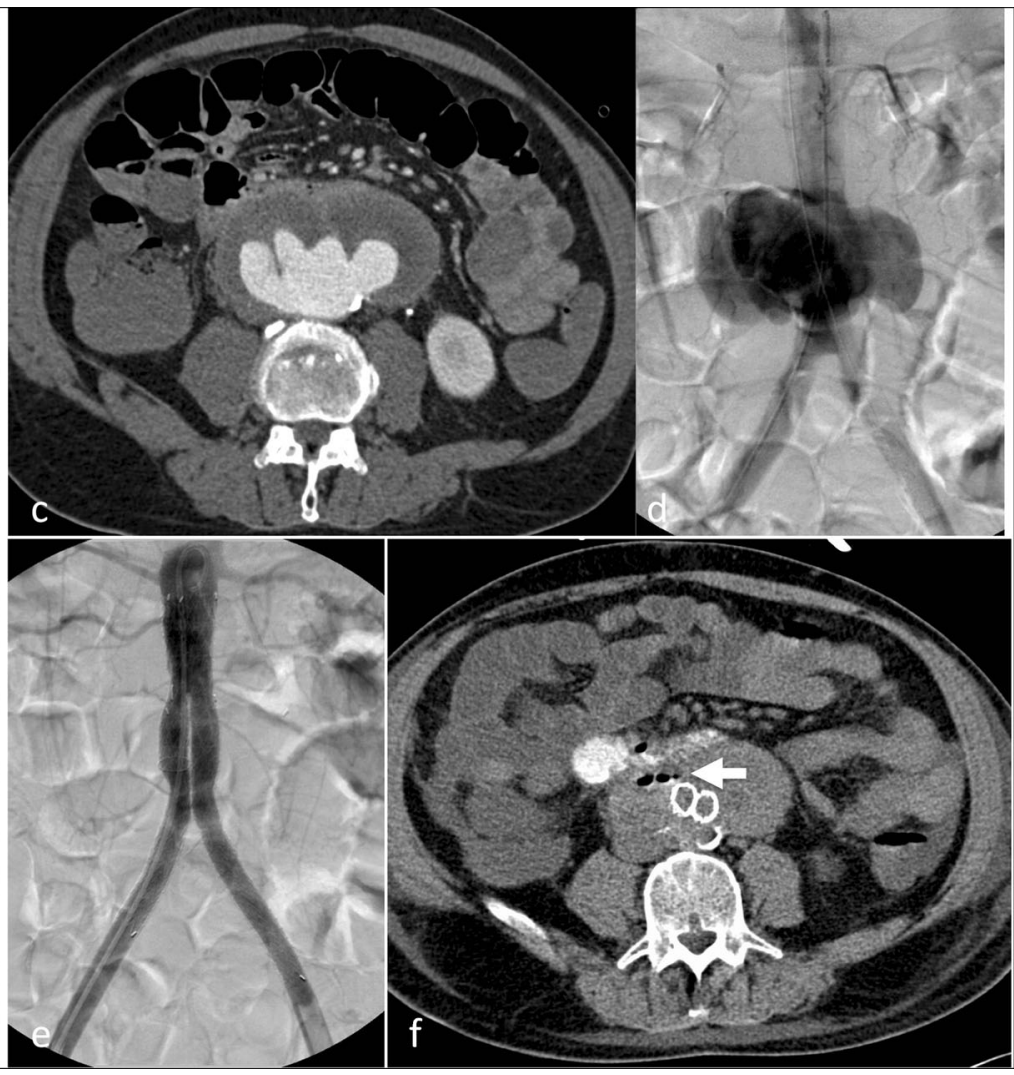

aneurysm. He had previous aortic repair (15 years ago) and had also been suffering from intermittent haematemesis for 3 months. CTA (c) and DSA (d) demonstrated an aneurysm of the distal anastomosis in close proximity to the duodenum. A bifurcated stent graft was implanted (e). The aneurysm was completely excluded. A follow-up CT on the next day (after oral contrast) nicely demonstrated the aorto-duodenal fistulae (arrow) (f) 
Fig. 12 Iatrogenic aortic injury. A 36-year-old female patient underwent laparoscopic removal of an ovarian cyst; $6 \mathrm{~h}$ later she presented with hypotension and severe abdominal pain. CTA [(a) axial section, arrowhead; (b) coronal reformation; (c) volumerendered image, arrow] demonstrated a left lateral laceration of the aorta, confirmed to be a trocar injury during vascular surgical reconstruction. A 6-month follow-up CT demonstrates a rather smooth aortic contour at the level of the original injury (d arrowhead). A 66-year-old patient presented with an aneurysm of the coeliac trunk/common hepatic artery (e). During an endovascular approach for stent graft implantation, when the Simmons type II catheter was configured in the aortic arch, the patient reported severe thoracic pain; DSA (f) and CTA [(g-h) on a single slice CT scanner] confirmed the suspicion of a type A aortic dissection with a true lumen (arrowhead) and false lumen (arrows) on unsubtracted angiographic images
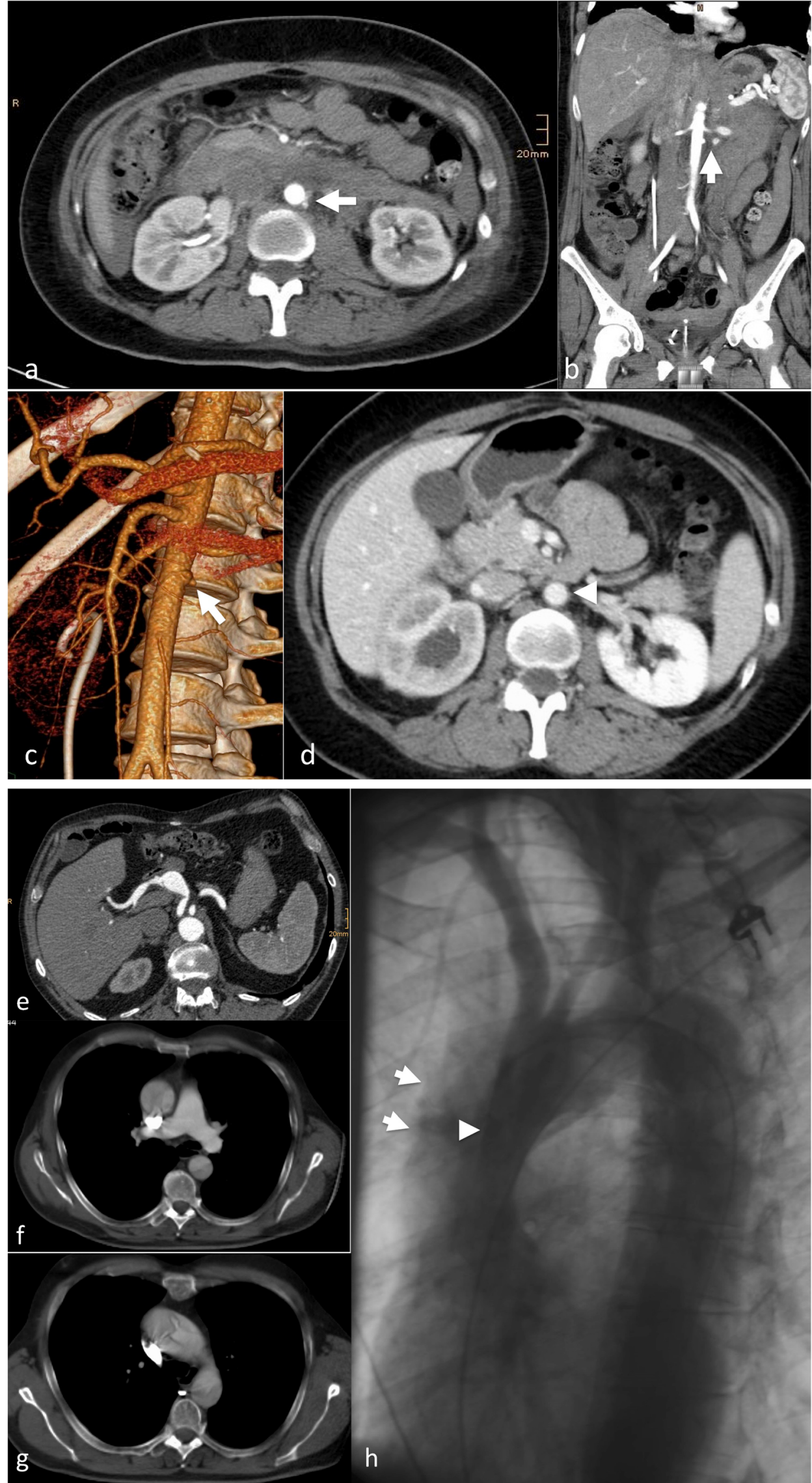
origin. Demonstration of contrast extravasation from the aorta to the duodenum is diagnostic of an acute bleeding aortoenteric fistula. Therapy consists of an urgent individual interdisciplinary approach, potentially combining EVAR for bridging and open surgery as the definitive treatment approach (Fig. 11).

\section{Iatrogenic aortic injury}

There are various mechanisms for iatrogenic aortic injury. On one hand it can be caused by direct external injury from surgical procedures, causing direct bleeding or (delayed) pseudoaneurysm. If detected intraoperatively, it can be treated by a direct surgical approach. Delayed bleeding or pseudoaneurysm formation is usually detected by CTA or by surgical revision (Fig. 12a-d). On the other hand it may be caused by an endovascular approach. The larger the catheters or introduction materials (sheaths, etc.) are, the higher the number of access (= iliac) vessel complications (rupture, dissection, occlusion), which are by far the most common complications [37]. With the introduction of stent grafting, endovascular aortic valve implantations and aortic balloon pumps, the numbers of direct aortic injuries have increased, albeit not by much. Dissections are the most common type of injuries, but they are not exclusively caused by large-calibre devices; they can also be caused by small catheters (Fig. 12eh).

Retrograde aortic (type A) dissections have been reported as complications of stent graft implantation for the treatment of type B dissections or descending aortic aneurysms in the literature [38] and may also be considered an iatrogenic injury.

\section{Summary}

Aortic emergencies are relatively rare, but they have to be detected very quickly. CTA is the imaging method of choice and helps to decide whether elective, urgent or emergent treatment is necessary. EVAR and surgical repair are the treatment approaches of choice.

Open Access This article is distributed under the terms of the Creative Commons Attribution License which permits any use, distribution, and reproduction in any medium, provided the original author(s) and the source are credited.

\section{References}

1. Knaut AL, Cleveland JC Jr (2003) Aortic emergencies. Emerg Med Clin North Am 21(4):817-845
2. Go AS, Mozaffarian D, Roger VL et al (2013) Heart disease and stroke statistics-2013 update: a report from the American Heart Association. Circulation 127(1):e6-e245

3. Authors/Task Force m, Erbel R, Aboyans V et al (2014) 2014 ESC guidelines on the diagnosis and treatment of aortic diseases: document covering acute and chronic aortic diseases of the thoracic and abdominal aorta of the adult. The task force for the diagnosis and treatment of aortic diseases of the European Society of Cardiology (ESC). Eur Heart J 35(41):2873-2926

4. Hiratzka LF, Bakris GL, Beckman JA et al (2010) 2010 ACCF/AHA/ AATS/ACR/ASA/SCA/SCAI/SIR/STS/SVM guidelines for the diagnosis and management of patients with thoracic aortic disease: a report of the American College of Cardiology Foundation/American Heart Association Task Force on Practice Guidelines, American Association for Thoracic Surgery, American College of Radiology, American Stroke Association, Society of Cardiovascular Anesthesiologists, Society for Cardiovascular Angiography and Interventions, Society of Interventional Radiology, Society of Thoracic Surgeons, and Society for Vascular Medicine. Circulation 121(13):e266-369

5. Fattori R, Cao P, De Rango P et al (2013) Interdisciplinary expert consensus document on management of type $\mathrm{B}$ aortic dissection. $\mathrm{J}$ Am Coll Cardiol 61(16):1661-1678

6. Chin AS, Fleischmann D (2012) State-of-the-art computed tomography angiography of acute aortic syndrome. Semin Ultrasound CT MR 33(3):222-234

7. Moll FL, Powell JT, Fraedrich G et al (2011) Management of abdominal aortic aneurysms clinical practice guidelines of the European society for vascular surgery. Eur J Vasc Endovasc Surg Off J Eur Soc Vasc Surg 41(Suppl 1):S1-S58

8. Azizzadeh A, Ray HM, Dubose JJ et al (2014) Outcomes of endovascular repair for patients with blunt traumatic aortic injury. $\mathrm{J}$ Trauma Acute Care Surg 76(2):510-516

9. Estrera AL, Miller CC 3rd, Guajardo-Salinas G et al (2013) Update on blunt thoracic aortic injury: fifteen-year single-institution experience. J Thorac Cardiovasc Surg 145(3 Suppl):S154-158

10. Forman MJ, Mirvis SE, Hollander DS (2013) Blunt thoracic aortic injuries: CT characterisation and treatment outcomes of minor injury. Eur Radiol 23(11):2988-2995

11. Kitai T, Kaji S, Yamamuro A et al (2011) Detection of intimal defect by 64-row multidetector computed tomography in patients with acute aortic intramural hematoma. Circulation 124(11 Suppl):S174-178

12. Azizzadeh A, Keyhani K, Miller CC 3rd, Coogan SM, Safi HJ, Estrera AL (2009) Blunt traumatic aortic injury: initial experience with endovascular repair. J Vasc Surg 49(6):1403-1408

13. Lee WA, Matsumura JS, Mitchell RS et al (2011) Endovascular repair of traumatic thoracic aortic injury: clinical practice guidelines of the Society for Vascular Surgery. J Vasc Surg 53(1):187-192

14. Osgood MJ, Heck JM, Rellinger EJ et al (2014) Natural history of grade I-II blunt traumatic aortic injury. J Vasc Surg 59(2):334-341

15. Starnes BW, Lundgren RS, Gunn M et al (2012) A new classification scheme for treating blunt aortic injury. J Vasc Surg 55(1):47-54

16. Antonello M, Menegolo M, Maturi C et al (2013) Intentional coverage of the left subclavian artery during endovascular repair of traumatic descending thoracic aortic transection. J Vasc Surg 57(3):684-690, e681

17. Iezzi R, Dattesi R, Pirro F et al (2011) CT angiography in stent-graft sizing: impact of using inner vs. outer wall measurements of aortic neck diameters. J Endovasc Ther Off J Int Soc Endovasc Specialists 18(3):280-288

18. Jonker FH, van Keulen JW, Schlosser FJ et al (2011) Thoracic aortic pulsatility decreases during hypovolemic shock: implications for stent-graft sizing. J Endovasc Ther Off J Int Soc Endovasc Specialists 18(4):491-496

19. Rimon U, Shinfeld A, Gayer G (2014) Traumatic injury of the thoracic aorta treated with stent-graft: is long-term CT angiography follow-up justified? Clin Radiol 69(5):e207-210 
20. Rasche V, Oberhuber A, Trumpp S et al (2011) MRI assessment of thoracic stent grafts after emergency implantation in multi trauma patients: a feasibility study. Eur Radiol 21(7):1397-1405

21. Vilacosta I, Roman JA (2001) Acute aortic syndrome. Heart 85(4): 365-368

22. Castaner E, Andreu M, Gallardo X, Mata JM, Cabezuelo MA, Pallardo Y (2003) CT in nontraumatic acute thoracic aortic disease: typical and atypical features and complications. Radiographics: a review publication of the Radiological Society of North America, Inc, 23 Spec No:S93-110. doi: 10.1148/rg.23si035507

23. Dake MD, Thompson M, van Sambeek M, Vermassen F, Morales JP, Investigators D (2013) DISSECT: a new mnemonic-based approach to the categorization of aortic dissection. J Endovasc Ther Off J Int Soc Endovasc Specialists 46(2):175-190

24. Evangelista A, Mukherjee D, Mehta RH et al (2005) Acute intramural hematoma of the aorta: a mystery in evolution. Circulation 111(8): 1063-1070

25. Eggebrecht H, Plicht B, Kahlert P, Erbel R (2009) Intramural hematoma and penetrating ulcers: indications to endovascular treatment. $\mathrm{J}$ Endovasc Ther Off J Int Soc Endovasc Specialists 38(6):659-665

26. Ganaha F, Miller DC, Sugimoto K et al (2002) Prognosis of aortic intramural hematoma with and without penetrating atherosclerotic ulcer: a clinical and radiological analysis. Circulation 106(3):342-348

27. Coady MA, Rizzo JA, Elefteriades JA (1999) Pathologic variants of thoracic aortic dissections. Penetrating atherosclerotic ulcers and intramural hematomas. Cardiol Clin 17(4):637-657

28. Pauls S, Orend KH, Sunder-Plassmann L, Kick J, Schelzig H (2007) Endovascular repair of symptomatic penetrating atherosclerotic ulcer of the thoracic aorta. J Endovasc Ther Off J Int Soc Endovasc Specialists 34(1):66-73

29. Vu KN, Kaitoukov Y, Morin-Roy F et al (2014) Rupture signs on computed tomography, treatment, and outcome of abdominal aortic aneurysms. Insights into Imaging 5(3):281-293
30. Rakita D, Newatia A, Hines JJ, Siegel DN, Friedman B (2007) Spectrum of CT findings in rupture and impending rupture of abdominal aortic aneurysms. Radiographics a Rev Publ Radiol Soc N Am Inc 27(2):497-507

31. Hamilton H, Constantinou J, Ivancev K (2014) The role of permissive hypotension in the management of ruptured abdominal aortic aneurysms. J Cardiovasc Surg 55(2):151-159

32. Investigators IT, Powell JT, Sweeting MJ et al (2014) Endovascular or open repair strategy for ruptured abdominal aortic aneurysm: 30 day outcomes from IMPROVE randomised trial. BMJ 348:f7661

33. Kan CD, Lee HL, Luo CY, Yang YJ (2010) The efficacy of aortic stent grafts in the management of mycotic abdominal aortic aneurysm-institute case management with systemic literature comparison. Ann Vasc Surg 24(4):433-440

34. Yu SY, Hsieh HC, Ko PJ, Huang YK, Chu JJ, Lee CH (2011) Surgical outcome for mycotic aortic and iliac anuerysm. World J Surg 35(7):1671-1678

35. Saratzis N, Saratzis A, Melas N, Ktenidis K, Kiskinis D (2008) Aortoduodenal fistulas after endovascular stent-graft repair of abdominal aortic aneurysms: single-center experience and review of the literature. J Endovasc Ther Off J Int Soc Endovasc Specialists 15(4):441-448

36. Vu QD, Menias CO, Bhalla S, Peterson C, Wang LL, Balfe DM (2009) Aortoenteric fistulas: CT features and potential mimics. Radiographics a Rev Publ Radiol Soc N Am Inc 29(1):197-209

37. Genereux P, Webb JG, Svensson LG et al (2012) Vascular complications after transcatheter aortic valve replacement: insights from the PARTNER (Placement of AoRTic TraNscathetER Valve) trial. J Am Coll Cardiol 60(12):1043-1052

38. Kaji S, Akasaka T, Katayama M et al (2003) Prognosis of retrograde dissection from the descending to the ascending aorta. Circulation 108(Suppl 1):II300-306 